\title{
Field-Induced Gap in the Spin-1/2 Heisenberg Chain Compound Cu-Pyrimidine Dinitrate: ESR Studies in Magnetic Fields up to $63 \mathrm{~T}$
}

\author{
M. Ozerov $\cdot$ J. Wosnitza $\cdot$ E. Čižmár • \\ R. Feyerherm • S.R. Manmana • F. Mila • \\ S.A. Zvyagin
}

Received: 6 June 2012 / Accepted: 17 September 2012 / Published online: 27 September 2012

(C) Springer Science+Business Media New York 2012

\begin{abstract}
Results of electron spin resonance studies of copper pyrimidine dinitrate $\left(\mathrm{Cu}-\mathrm{PM},\left[\mathrm{PM}-\mathrm{Cu}\left(\mathrm{NO}_{3}\right)_{2}\left(\mathrm{H}_{2} \mathrm{O}\right)_{2}\right]_{n}, \mathrm{PM}=\right.$ pyrimidine $)$, a spin- $1 / 2$ antiferromagnetic chain material with alternating $g$-tensor and Dzyaloshinskii-Moriya interactions, in pulsed magnetic fields up to $63 \mathrm{~T}$ are reported. The field-induced gap shows a nonmonotonic behavior with a minimum in the vicinity of the saturation field $H_{\text {sat }}=$ 48.5 T. This is associated with a transition from the sine-Gordon region to a spinpolarized state with magnon excitations. By comparing the entire set of experimental data with results of density matrix renormalization group calculations for $\mathrm{Cu}-\mathrm{PM}$, the validity of the used theoretical approach is proven.
\end{abstract}

Keywords Electron spin resonance $\cdot$ Field-induced gap $\cdot$ Solitons $\cdot$ Breathers

\footnotetext{
M. Ozerov $(\bowtie) \cdot$ J. Wosnitza · S.A. Zvyagin

Dresden High Magnetic Field Laboratory (HLD), Helmholtz-Zentrum Dresden-Rossendorf, 01328

Dresden, Germany

e-mail:m.ozerov@hzdr.de

E. Čižmár

Centre of Low Temperature Physics, P.J. Šafárik University, 04154 Košice, Slovakia

R. Feyerherm

Helmholtz-Zentrum Berlin für Materialien und Energie, 14109 Berlin, Germany
}

S.R. Manmana · F. Mila

Institute of Theoretical Physics, Ecole Polytechnique Fédérale de Lausanne, 1015 Lausanne,

Switzerland

S.R. Manmana

JILA, Department of Physics, University of Colorado, 440 UCB, Boulder, CO 80309, USA

Springer 


\section{Introduction}

Low-dimensional quantum magnets serve as model systems for investigating numerous fascinating phenomena in materials with cooperative ground states, in particular, induced by magnetic field. A Heisenberg antiferromagnetic (AF) spin-1/2 chain with exchange interaction $J$ and Dzyaloshinskii-Moriya (DM) interaction (or alternating $g$-tensor) in a field $H$ can be mapped to a simple Heisenberg chain with a staggered transverse field $h \propto H$, described by the effective spin Hamiltonian

$$
\mathcal{H}=\sum_{j}\left[J \mathbf{S}_{j} \cdot \mathbf{S}_{j+1}-H S_{j}^{z}-h(-1)^{j} S_{j}^{x}\right] .
$$

The sine-Gordon quantum field theory [1-4] predicts that the magnetic excitation spectrum in magnetic fields $H<J / g \mu_{B}$ is to be formed by a soliton and multiple bound states (breathers). An energy gap, $\Delta \propto H^{2 / 3}$, can be observed directly by means of electron spin resonance (ESR) [5, 6] and is formed by the first breather mode.

Numerical simulations based on the density matrix renormalization group (DMRG) calculations by Zhao et al. [7] have shown that when magnetic field approaches the magnetization saturation phase the energy gap has a non-monotonic energy vs field dependence, exhibiting a minimum around $H_{s a t}$. Using field-theory arguments, Fouet et al. [8] showed that the gap around the saturation field scales as $h^{4 / 5}$.

Such remarkable changes in the excitation spectrum in the vicinity of the saturation field have been identified experimentally first in copper benzoate [6]. A nonmonotonic behavior with a minimum in the vicinity of the saturation field has been revealed in the excitation spectrum of this compound.

Recently, a signature of soliton and three breathers, as well as specific temperature and field dependencies of ESR parameters (linewidth and $g$-factor) have been observed in the $\mathrm{S}=1 / 2$ AF chain material copper pyrimidine dinitrate $\left(\left[\mathrm{PM}-\mathrm{Cu}\left(\mathrm{NO}_{3}\right)_{2}\left(\mathrm{H}_{2} \mathrm{O}\right)_{2}\right]_{n}, \mathrm{PM}=\right.$ pyrimidine; hereafter $\left.\mathrm{Cu}-\mathrm{PM}\right)$ [9] in magnetic fields up to $25 \mathrm{~T}$, beautifully confirming the applicability of the sine-Gordon quantum-field theoretical approach $[5,10]$ for $H<J / g \mu_{B}$. Here, we report on ESR studies of the field-induced gap behavior in magnetic fields up to $63 \mathrm{~T}$. A pronounced change in the frequency-field dependence of the lowest-energy mode has been observed in the vicinity of the saturation field, $H_{\text {sat }}=48.5 \mathrm{~T}$ [11], clearly indicating a crossover from the soliton-breather to the magnon state. The data are compared with numerically exact DMRG results.

\section{Experimental Details}

High-field ESR experiments of Cu-PM were performed at the Dresden High Magnetic Field Laboratory (Hochfeld-Magnetlabor Dresden, HLD) using a pulsed-field ESR spectrometer [12] equipped with tunable sources of millimeter-wave radiation (product of Virginia Diodes Inc.). Measurements were done using a transmission-type probe in Faraday configuration. A $8.5 \mathrm{MJ} / 70 \mathrm{~T}$ magnet was employed to generate 
pulsed magnetic fields with a pulse-field rise time of $35 \mathrm{~ms}$ and full-pulse duration of about $150 \mathrm{~ms}$. The magnetic field was applied along the $c^{\prime \prime}$ direction, which is characterized by the maximal value of the staggered magnetization for $\mathrm{Cu}-\mathrm{PM}$ [9]. Experiments were performed at a temperature of $1.9 \mathrm{~K}$. High-quality single-crystals of $\mathrm{Cu}$ PM with typical size of $3 \times 3 \times 0.5 \mathrm{~mm}^{3}$ were used. 2,2-Diphenyl-1-Picrylhydrazyl (known as DPPH) was employed for the calibration of the magnetic field. In our experiments, magnetic field was measured with the accuracy better than $1 \%$.

\section{Results and Discussion}

An example of ESR spectrum obtained at a frequency of $295.2 \mathrm{GHz}$ is shown in Fig. 1. The field dependencies of the ESR magnetic excitations observed in pulsed magnetic field up to $63 \mathrm{~T}$ are shown in Fig. 2 by symbols, while dash and solid lines correspond to results of the quantum-field sine-Gordon theory [5] and DMRG calculations, respectively.

According to the sine-Gordon quantum field theory for quantum spin-1/2 AF chains $[1,3,4]$, the field-induced energy gap can be calculated using the expression for the first breather excitation mode [3, 4]:

$$
\Delta_{g}=2 J \frac{2 \Gamma\left(\frac{\xi}{2}\right) v_{F}}{\sqrt{\pi} \Gamma\left(\frac{1+\xi}{2}\right)}\left[\frac{g \mu_{B} H}{J v_{F}} \frac{\pi \Gamma\left(\frac{1}{1+\xi}\right) c A_{x}}{2 \Gamma\left(\frac{\xi}{1+\xi}\right)}\right]^{\frac{1+\xi}{2}} \sin (\pi \xi / 2) .
$$

In this expression, $c$ is the proportionality coefficient connecting the uniform applied field $H$ and the effective staggered field $h=c H$ ( $c=0.083$ for Cu-PM [10]), the parameter $\xi=\left(2 /\left(\pi R^{2}\right)-1\right)^{-1}$, where $R$ is the compactification radius, and $v_{F}$ has

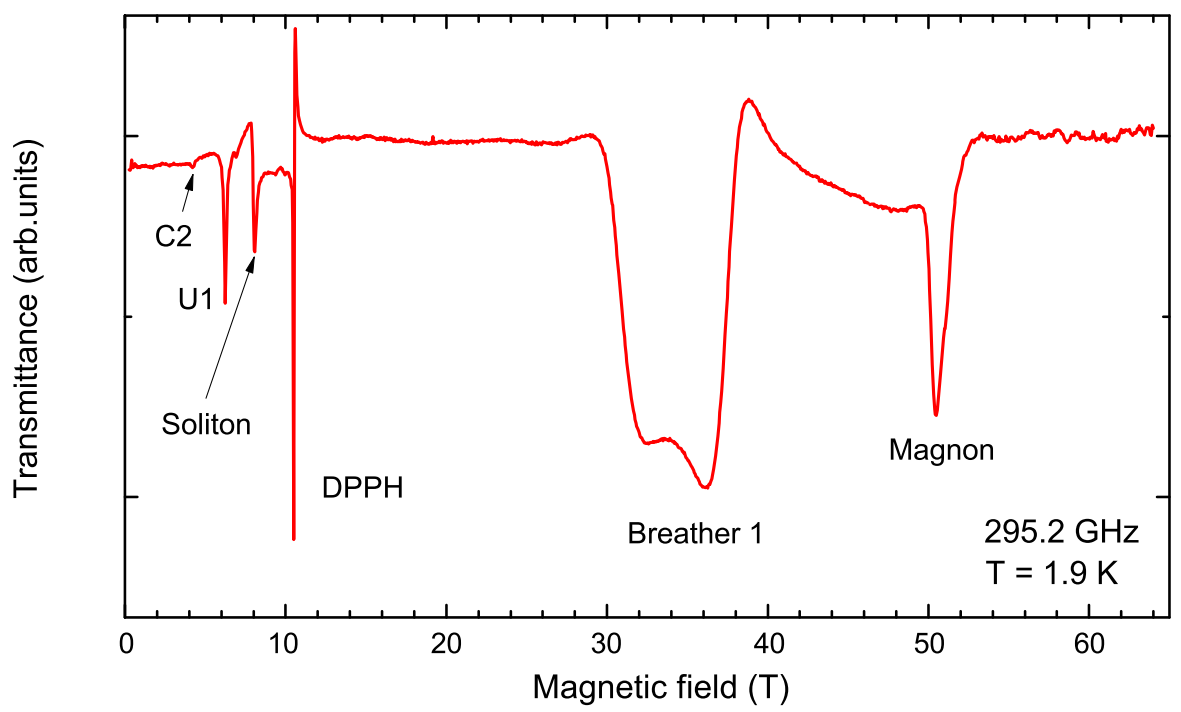

Fig. 1 ESR spectrum obtained at a frequency of $295.2 \mathrm{GHz}$ (Color figure online) 


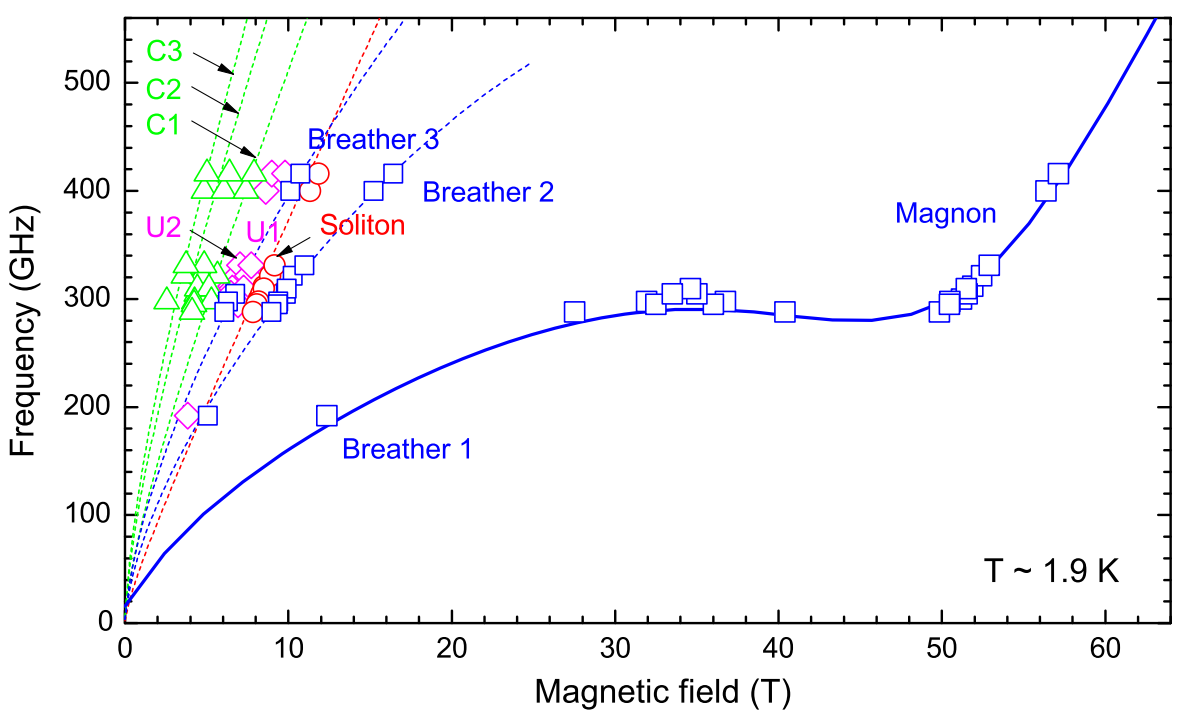

Fig. 2 Frequency-field dependencies of magnetic excitations in Cu-PM. Symbols denote experimental results, while dash and solid lines correspond to results of the quantum-field sine-Gordon theory [5] and DMRG calculations, respectively. Soliton excitations are denoted by circles; squares correspond to three breather and magnon modes; triangulares correspond to the edges of the soliton-breather continua $S B_{1}-S B_{3}$ (modes $C 1-C 3$, respectively); modes $U 1$ and $U 2$ (the experimental data are denoted by diamonds) correspond to the boundary bound state transitions [14] (Color figure online)

the meaning of the Fermi velocity $[3,4]$. The amplitude $A_{x}$, which is also a function of $H$, has recently been computed numerically [13]. Excellent agreement between the experimental data up to $25 \mathrm{~T}$ and the calculation using Eq. (2) was found [5].

Since the expression (2) is valid only up to $H \sim J / g \mu_{B}$, it is necessary to turn to another approach to calculate the spin gap in the microscopic model of Eq. (1) at higher fields $\left(H>J / g \mu_{B}\right)$. To this end, DMRG calculations for the model of Eq. (1) have been performed. The results of the DMRG calculations for the field-induced gap using the microscopic model described by Eq. (1) with $L=200$ lattice sites and $c=0.083$ [10] are shown in Fig. 2 by a solid line. These results were obtained by performing up to $10 \mathrm{DMRG}$ sweeps and keeping up to $m=500$ states, resulting in a discarded weight $\varepsilon \ll 10^{-10}$. Note that due to the presence of the transversefield term in the Hamiltonian (1), $S_{\text {total }}^{z}$ is not a good quantum number, limiting the possible system sizes. However, already for the system size shown, the results for the gap are essentially the same as in the thermodynamic limit, as revealed by comparing with smaller systems. Note, that the results of DMRG calculations agree well with experimental data up to $H_{s a t}$ and above. This establishes that the microscopic model of Eq. (1) provides a quantitative description of the spin-gap behavior in spin-1/2 sine-Gordon chains for all field values.

Let us comment on the physical mechanism behind the non-monotonic increase of the gap [7, 8]. In the absence of a staggered field, the system is gapless below $H=H_{\text {sat }}$, and it is in the fully spin-polarized state above. In that state, elementary excitations are magnons, and the gap opens linearly with $H-H_{s a t}$. The presence of a 
staggered field perpendicular to the external field opens a gap in the spectrum breaking the rotational symmetry around the field. Now, the impact of the staggered field is related to the magnitude of the induced transverse magnetization. Close to saturation, the spins are almost polarized, and the system cannot develop a large transverse staggered magnetization. So the staggered field is less efficient to open a gap close to saturation than at low field. This is the basic mechanism behind the different scalings of the gap with $c$ at low field $\left(\Delta \propto c^{2 / 3}\right)$ and close to saturation $\left(\Delta \propto c^{4 / 5}\right)$. For small enough $c$, this leads to a minimum of the gap around the saturation field. This explains the small but still well-resolved dip in the frequency-field dependence of the magnetic excitations in $\mathrm{Cu}-\mathrm{PM}$ in the vicinity of $H_{\text {sat }}$. Such a behavior appears to be a general feature of the high-field excitation spectrum of quantum spin-1/2 chain systems with alternating $g$-tensor and/or Dzyaloshonskii-Moriya interactions. More details of the ESR excitation spectrum in Cu-PM is given in Ref. [15].

\section{Conclusion}

We have presented detailed ESR studies of the field-induced dependence of the magnetic-excitation gap in Cu-PM, a material containing $S=\frac{1}{2}$ AF chains with alternating $g$-tensor and Dzyaloshinskii-Moriya interaction, in magnetic fields up to 63 T. The field-induced change in the gap behavior was observed directly, clearly indicating the effect of a suppression of the soliton-breather regime by a strong magnetic field followed by a transition into the fully spin-polarized phase with magnons as elementary excitations. By comparing the entire set of data with results of DMRG calculations the validity of the used theoretical approach has been proven. Our results are relevant for the understanding of the spin dynamics in copper benzoate and other $S=1 / 2$ Heisenberg AF chain systems.

Acknowledgements The authors would like to thank A.K. Kolezhuk for fruitful discussions. This work was partly supported by the Deutsche Forschungsgemeinschaft and EuroMagNET (EU contract No. 228043). E.C. appreciates the financial support by VEGA 1/0078/09 and APVV-0132-11, and S.R.M. acknowledges the support by PIF-NSF (grant No. 0904017). F.M. acknowledges the support of the Swiss National Fund and of MaNEP.

\section{References}

1. M. Oshikawa, I. Affleck, Phys. Rev. Lett. 79, 2883 (1997)

2. F.H.L. Essler, Phys. Rev. B 59, 14376 (1999)

3. I. Affleck, M. Oshikawa, Phys. Rev. B 60, 1038 (1999)

4. I. Affleck, M. Oshikawa, Phys. Rev. B 62, 9200 (2000)

5. S.A. Zvyagin, A.K. Kolezhuk, J. Krzystek, R. Feyerherm, Phys. Rev. Lett. 93, 027201 (2004)

6. H. Nojiri, A. Ajiro, T. Asano, J.-P. Boucher, New J. Phys. 8, 218 (2006)

7. J.Z. Zhao, X.Q. Wang, T. Xiang, Z.B. Su, L. Yu, Phys. Rev. Lett. 90, 207204 (2003)

8. J.-B. Fouet, O. Tchernyshyov, F. Mila, Phys. Rev. B 70, 174427 (2004)

9. R. Feyerherm, S. Abens, D. Günther, T. Ishida, M. Meißner, M. Meschke, T. Nogami, M. Steiner, J. Phys. Condens. Matter 12, 8495 (2000)

10. S.A. Zvyagin, A.K. Kolezhuk, J. Krzystek, R. Feyerherm, Phys. Rev. Lett. 95, 017207 (2005)

11. A.U.B. Wolter, H. Rakoto, M. Costes, A. Honecker, W. Brenig, A. Klümper, H.-H. Klauss, F.J. Litterst, R. Feyerherm, D. Jérome, S. Süllow, Phys. Rev. B 68, 220406(R) (2003) 
12. S.A. Zvyagin, M. Ozerov, E. Čižmár, D. Kamenskyi, S. Zherlistyn, T. Herrmannsdörfer, J. Wosnitza, R. Wünsch, W. Seidel, Rev. Sci. Instrum. 80, 073102 (2009)

13. F.H.L. Essler, A. Furusaki, T. Hikihara, Phys. Rev. B 68, 064410 (2003)

14. S.C. Furuya, M. Oshikawa, arXiv:1112.1088

15. S.A. Zvyagin, E. Čižmár, M. Ozerov, J. Wosnitza, R. Feyerherm, S.R. Manmana, F. Mila, Phys. Rev. B 83, 060409(R) (2011) 